\title{
USING EXPERT OPINIONS IN BAYESIAN ESTIMATION OF SYSTEM RELIABILITY*
}

\author{
BENT NATVIG, University of Oslo
}

\begin{abstract}
In this paper combining the opinions of $k$ experts about the reliabilities of $n$ components of a binary system is considered. Especially the case $n=2$ is treated in detail. This work generalizes papers by Huseby $(1986,1988)$ on the single component case. Since the experts often share data, he argues that their assessments will typically be dependent and that this difficulty cannot be handled without making judgements concerning the underlying sources of information and to what extent these are available to each of the experts. In the former paper the information available to the experts is modeled as a set of observations $Y_{1}, \cdots, Y_{m}$. These observations are then reconstructed as far as possible from the information provided by the experts and used as a basis for the combined judgement. This is called the retrospective approach. In the latter paper, the uncertain quantity is modeled as a future observation, $Y$, from the same distribution as the $Y_{i}$ 's. This is called the predictive approach. For the case, $n>1$, where each expert is giving opinions about more than one component, additional dependencies between the reliabilities of the components come into play. This is for instance true if two or more components are of similar type, are sharing a common environment or are exposed to common cause failures. In the generalized retrospective approach the joint prior distribution of the reliabilities is arrived at. When this is $M T P_{2}$ (Multivariate Totally Positive of Order 2), it is shown that the machinary of Natvig and Eide (1987) can be applied to arrive at the posterior distribution of system reliability, based on data both on the component and system level. Hence a key question to be answered is the following. When does the joint prior distribution of the reliabilities based on expert opinions in fact possess the $M T P_{2}$ property? A partial answer to this question is given.
\end{abstract}

\section{Introduction}

Consider, for a fixed point of time, $t$, a binary system of $n$ binary components. Let $(i=1, \cdots, n)$ :

$$
\begin{aligned}
& X_{i}= \begin{cases}1 & \text { if the ith component functions } \\
0 & \text { otherwise, }\end{cases} \\
& \underline{X}=\left(X_{1}, \cdots, X_{n}\right), \\
& \phi(\underline{X})= \begin{cases}1 & \text { if the system functions } \\
0 & \text { otherwise. }\end{cases}
\end{aligned}
$$

* To be presented at the Course-Congress on Reliability and Decision Making, Siena, Italy, October 15-26 1990. This research is part of the Nordic Terotechnology program supported by Nordisk Industrifond and NTNF. 
Let furthermore:

$$
\begin{aligned}
& E\left(X_{i} \mid p_{i}\right)=p_{i}=\text { the reliability of the ith component, } \\
& E(\phi(X) \mid h)=h=\text { the reliability of the system. }
\end{aligned}
$$

If we assume that $X_{1}, \cdots, X_{n}$ are independent given $p=\left(p_{1}, \cdots, p_{n}\right)$, we write:

$$
h=E(\phi(X) \mid p)=h(p)
$$

In Natvig and Eide (1987) it was assumed that the joint prior distribution of the reliabilities, before running any experiments on the component level, $\pi(p)$, can be written as:

$$
\pi(\underline{p})=\prod_{i=1}^{n} \pi_{i}\left(p_{i}\right),
$$

where $\pi_{i}\left(p_{i}\right)$ is the prior marginal distribution of $p_{i}$, i.e. we assumed that the components have independent prior reliabilities.

In this paper we assume that $k$ experts will provide the information about the reliabilities of the components. This work generalizes papers by Huseby $(1986,1988)$ on the single component case. Since the experts often share data, he argues that their assessments will typically be dependent and that this difficulty cannot be handled without making judgements concerning the underlying sources of information and to what extent these are available to each of the experts. In the former paper the information available to the experts is modeled as a set of observations $Y_{1}, \cdots, Y_{m}$. These observations are then reconstructed as far as possible from the information provided by the experts and used as a basis for the combined judgement of a decision maker (DM). This is called the retrospective approach. In the latter paper, the uncertain quantity is modeled as a future observation, $Y$, from the same distribution as the $Y_{i}$ 's. This is called the predictive approach.

For the case, $n>1$, where each expert is giving opinions about more than one component, additional dependencies between the reliabilities of the components come into play. This is for instance true if two or more components are of similar type, are sharing a common environment or are exposed to common cause failures. In the case of $X_{1}, \cdots, X_{n}$ independent given $p$, and the lifetimes being exponentially distributed with unknown failure rates $\lambda_{1}, \cdots, \lambda_{n}$, this problem is considered by Lindley and Singpurwalla (1986). Then obviously:

$$
p_{i}=\exp \left(-\lambda_{i} t\right) \quad i=1, \cdots, n \text {. }
$$

In the latter paper the jth expert, $j=1, \cdots, k$, expresses his opinion about $\lambda_{i}$ and hence of $p_{i}$ in terms of a normal distribution for $\theta_{i}=\ln \lambda_{i}, i=1, \cdots, n$. He provides its mean $m_{j i}$ and standard deviation $s_{j i}$ but also $\rho_{j i r}$ being the personal correlation between $\theta_{i}$ and $\theta_{r}, j=1, \cdots, k ; i, r=1, \cdots, n, i \neq r$. In addition the DM has to provide his personal correlations between the $m_{j i}$ 's for fixed expert $j$ and different components, for fixed component $i$ and different experts and finally for both different experts and components. The great drawback of this approach is the difficulty of assessing these correlations directly without having an underlying model as in Huseby $(1986,1988)$. 
Lindley and Singpurwalla (1986) use an approximation technique suggested by Laplace, which has been pointed out to be quite good by Tierney and Kadane (1984), to arrive at the corresponding uncertainty in $h(p)$ for a parallell system of independent components. They claim that the results may easily be generalized to cover any coherent system of independent components. This is not true since representing a coherent system of independent components by a series-parallel structure introduces replicated components which of course are dependent. For details see the excellent textbook Barlow and Proschan (1975).

In Section 2 and 3 of the present paper the case $n=2$ is treated in detail. The generalized predictive approach is treated in the former section. Here the uncertain quantities $\left(Z_{1}, Z_{2}\right)$ are the lifetimes of the two components. These are assumed to have a bivariate exponential distribution. In the latter section the generalized retrospective approach is considered. For the fixed point of time, $t$, let:

$$
\begin{aligned}
& r_{i}=P[\text { only the ith component functions], } i=1,2 \\
& r_{3}=P[\text { both component functions]. }
\end{aligned}
$$

Then obviously:

$$
p_{1}=r_{1}+r_{3} \quad, p_{2}=r_{2}+r_{3} .
$$

Based on expert opinions the joint prior distribution $\pi\left(p_{1}, p_{2}\right)$ is arrived at.

In Section 4 it is shown, for general $n$, that when $\pi(p)$ is $M T P_{2}$ (Multivariate Totally Positive of Order 2), then the machinary of Natvig and Eide (1987) can be applied to arrive at the posterior distribution of system reliability, based on data both an the component and system level. Hence a key question to be answered is the following. When does $\pi(p)$ based on expert opinions in fact possess the $M T P_{2}$ property? For the case treated in Section 3 this is shown to be true for the non trivial case where either $r_{1}=0$ or $r_{2}=0$.

\section{The predictive approach}

The deductions in this section follow the main lines of the example given in Section 3 of Huseby (1988). The jump from the univariate to the bivariate case, however, gives sufficient obstacles to overcome. Let $\left(Z_{1}, Z_{2}\right)$ be the lifetimes of the two components. The DM specifies a set of vectors of nonnegative numbers:

$$
z_{s}=\left(z_{11}, z_{21}, z_{12 s}, z_{22}, z_{13}, z_{23} s\right), s=1, \cdots, r .
$$

He then asks the jth expert to describe his uncertainty about $\left(Z_{1}, Z_{2}\right)$ by specifying a set of vectors of probabilities:

$$
\underline{\pi}_{j \bullet}=\left(\pi_{j 1 s}, \pi_{j 2 s}, \pi_{j 3 s}\right), s=1, \cdots, r
$$

such that:

$$
P\left(Z_{1}>z_{1 v s}, Z_{2}>z_{2 v s}\right)=\pi_{j v s}, v=1,2,3 ; s=1, \cdots, r .
$$


The DM then faces the problem of computing his posterior distribution for $\left(Z_{1}, Z_{2}\right)$ given $\underline{\pi}_{j s}, j=1, \cdots, k ; s=1, \cdots, r$.

We assume that the DM assesses that given the hyperparameters $\theta_{1}, \theta_{2}, \theta_{3},\left(Z_{1}, Z_{2}\right)$ and the imaginary observations $\left(Z_{1 i}, Z_{2 i}\right), i=1, \cdots, m$ are mutually independent with a bivariate exponential distribution of the Marshall-Olkin type, i.e.

$$
P\left(Z_{1}>z_{1}, Z_{2}>z_{2} \mid \theta_{1}, \theta_{2}, \theta_{3}\right)=\exp \left(-\theta_{1} z_{1}-\theta_{2} z_{2}-\theta_{3} z_{3}\right)
$$

where $z_{3}=\max \left(z_{1}, z_{2}\right)$. For details on the properties of this distribution we refer to Barlow and Proschan (1975). Furthermore, we assume that the prior distributions of $\theta_{l}, l=1,2,3$ both for the DM and the jth expert are independent gamma distributions with shape parameter and scale parameter respectively equal to $\left(a_{l}, b_{l}\right)$ for the DM and $\left(a_{j l}, b_{j l}\right)$ for the jth expert, $j=1, \cdots, k ; l=1,2,3$. In Huseby (1988) just a vague gamma distribution with parameters close to zero is used as a prior for the single parameter $\theta$.

As in the latter paper let $B_{0}, B_{1}, \cdots, B_{k}$ be disjoint subsets of the index set $\{1, \cdots, m\}$ such that:

$$
B_{0} \bigcup B_{1} \bigcup \cdots \bigcup B_{k}=\{1, \cdots, m\}
$$

It is then assessed that the jth expert has access to information on the $\left(Z_{1 i}, Z_{2 i}\right)$ 's with indices in the set $A_{j}=\left(B_{0} \bigcup B_{j}\right), j=1, \cdots, k$. Thus $B_{0}$ is the set of common information, while the $B_{j}$ 's are the sets of individual informations. As opposed to Huseby (1988) we express the information as survivals of the two components beyond specific time points; i.e.

$$
\left\{Z_{1 i}>z_{1 i}, Z_{2 i}>z_{2 i}\right\}, \quad i=1, \cdots, m
$$

instead of just observed lifetimes. This turns out to be at least mathematically advantageous.

Introduce:

$$
\begin{aligned}
z_{3 i} & =\max \left(z_{1 i}, z_{2 i}\right) \\
t_{j l} & =\sum_{i \in A_{j}} z_{l i} \quad, j=1, \cdots, k ; l=1,2,3 \\
t_{l} & =\sum_{i=1}^{m} z_{l i} \quad, l=1,2,3
\end{aligned}
$$


We now have by standard calculations involving Bayes theorem:

$$
\begin{aligned}
& P\left[\left(Z_{1}>z_{1}, Z_{2}>z_{2}\right) \mid \bigcap_{i=1}^{m}\left(Z_{1 i}>z_{1 i}, Z_{2 i}>z_{2 i}\right)\right] \\
& =k \int_{\theta_{1}=0}^{\infty} \int_{\theta_{2}=0}^{\infty} \int_{\theta_{3}=0}^{\infty} P\left(Z_{1}>z_{1}, Z_{2}>z_{2} \mid \theta_{1}, \theta_{2}, \theta_{3}\right) \\
& \cdot P\left[\bigcap_{i=1}^{m}\left(Z_{1 i}>z_{1 i}, Z_{2 i}>z_{2 i}\right) \mid \theta_{1}, \theta_{2}, \theta_{3}\right] \prod_{l=1}^{3} \frac{b_{l}^{a_{l}} \theta_{l}^{a_{l}-1}}{\Gamma\left(a_{l}\right)} \exp \left(-b_{l} \theta_{l}\right) d \theta_{1} d \theta_{2} d \theta_{3} \\
& =\prod_{l=1}^{3}\left(\frac{b_{l}+t_{l}}{b_{l}+t_{l}+z_{l}}\right)^{a_{l}}
\end{aligned}
$$

The constant $k$ is determined by noting that $z_{1}=z_{2}=0$ gives a joint survival probability of 1 . Similarly we get:

$$
\begin{aligned}
& \left.P\left[Z_{1}>z_{1}, Z_{2}>z_{2}\right) \mid \bigcap_{i \in A_{j}}\left(Z_{1 i}>z_{1 i}, Z_{2 i}>z_{2 i}\right)\right] \\
& =\prod_{l=1}^{3}\left(\frac{b_{j l}+t_{j l}}{b_{j l}+t_{j l}+z_{l}}\right)^{a_{j l}}
\end{aligned}
$$

Introducing $z_{3 v s}=\max \left(z_{1 v s}, z_{2 v s}\right), v=1,2,3 ; s=1, \cdots, r$, we get by combining $(2.1)$ and (2.3) the following $r$ sets of 3 equations to determine, for fixed $j=1, \cdots, k$, the information $t_{j l}, l=1,2,3$ :

$$
\prod_{l=1}^{3}\left(\frac{b_{j l}+t_{j l}}{b_{j l}+t_{j l}+z_{l v s}}\right)^{a_{j l}}=\pi_{j v s}, \quad v=1,2,3 ; s=1, \ldots, r
$$

Note that for fixed $s$ there is no guarantee that we end up by a unique solution to these 3 equations satisfying the obvious claim:

$$
t_{j 3} \geq \max \left(t_{j 1}, t_{j 2}\right) \geq \min \left(t_{j 1}, t_{j 2}\right) \geq 0
$$

Furthermore, even if all of the $r$ sets of equations give a unique, acceptable solution these will in general be different. Ideally we should calculate a posterior distribution for $t_{j l}, l=$ $1,2,3$ based on the assessments $\pi_{j v_{0}}, v=1,2,3 ; s=1, \cdots, r$. However, as an approximation we will at the present stage of research suggest as Huseby (1988) that one should base the subsequent calculations for fixed $j=1, \cdots, k$ and fixed $l=1,2,3$ on the averages of $t_{j l}$, for the sets of equations having a unique, acceptable solution.

A general investigation into the set of equations (2.4), for instance by a computer program performing algebraic manupulations, is outside the scope of the present paper. However, 
the DM can design his experiment in a clever way leading both to easier assessments for the experts and simpler calculations. For instance he can specify:

$$
\underline{z}_{s}=\left(z_{s}, z_{s}, z_{s}, 0,0, z_{s}\right), s=1, \cdots, r
$$

leading to rather easy assessments in (2.1). Let us furthermore for simplicity assume that the jth expert assesses:

$$
a_{j l}=a_{j 1}, \quad, j=1, \cdots, k ; l=2,3
$$

By introducing

$$
\begin{aligned}
& x_{j l}=b_{j l}+t_{j l} \\
& y_{j v s}=\left(\pi_{j v s}\right)^{a_{j 1}^{-1}}, j=1, \cdots, k ; v=1,2,3 ; s=1, \cdots, r,
\end{aligned}
$$

and then suppressing the indices $j$ and $s,(2.4)$ reduces to:

$$
\begin{aligned}
& x_{1} x_{2} x_{3}=y_{1}\left(x_{1}+z\right)\left(x_{2}+z\right)\left(x_{3}+z\right) \\
& x_{1} x_{2} x_{3}=y_{2}\left(x_{1}+z\right) x_{2}\left(x_{3}+z\right) \\
& x_{1} x_{2} x_{3}=y_{3} x_{1}\left(x_{2}+z\right)\left(x_{3}+z\right)
\end{aligned}
$$

Disregarding unacceptable solutions involving either $x_{1}=0, x_{2}=0$ or both, the system above is easily solved for the variables $x_{l} /\left(x_{l}+z\right), l=1,2,3$. This leads to the following solution:

$$
\begin{aligned}
& x_{1}=y_{1} z /\left(y_{3}-y_{1}\right) \\
& x_{2}=y_{1} z /\left(y_{2}-y_{1}\right) \\
& x_{3}=y_{2} y_{3} z /\left(y_{1}-y_{2} y_{3}\right)
\end{aligned}
$$

Hence we get $(j=1, \cdots, k ; s=1, \cdots, r)$ :

$$
\begin{aligned}
& t_{j 1}=\pi_{j 1 s}^{a_{j 1}^{-1}} \cdot z_{s} /\left(\pi_{j 3 s}^{a_{j 1}^{-1}}-\pi_{j 1 s}^{a_{j 1}^{-1}}\right)-b_{j 1} \\
& t_{j 2}=\pi_{j 1 s}^{a_{j 1}^{-1}} z_{s} /\left(\pi_{j 2 s}^{a_{j 1}^{-1}}-\pi_{j 1 s}^{a_{j 1}^{-1}}\right)-b_{j 2} \\
& t_{j 3}=\pi_{j 2 s}^{a_{j 1}^{-1}} \pi_{j 3 s}^{a_{j 1}^{-1}} z_{s}\left(\pi_{j 1 s}^{a_{j 1}^{-1}}-\pi_{j 2 s}^{a_{j 1}^{-1}} \pi_{j 3 s}^{a_{j 1}^{-1}}\right)-b_{j 3}
\end{aligned}
$$

Let us give some comments on when (2.10) satisfies the claim (2.5) of being a set of acceptable solutions.

A necessary condition for $t_{j 1}$ to be nonnegative is that:

$$
\pi_{j 3 s}=P\left(Z_{1}>0, Z_{2}>z_{s}\right) \geq P\left(Z_{1}>z_{s}, Z_{2}>z_{s}\right)=\pi_{j 1 s}
$$

which is always true. The same argument applies to $t_{j 2}$. Hence a necessary and sufficient condition for $\min \left(t_{j 1}, t_{j 2}\right)$ to be nonnegative is that

$$
\pi_{j 1 s}^{a_{j 1}^{-1}} z_{s} /\left(\pi_{j 4-l s}^{a_{j 1}^{-1}}-\pi_{j 10}^{a_{j 1}^{-1}}\right) \geq b_{j l}, l=1,2
$$


A necessary condition for $t_{j 3}$ to be nonnegative is that:

$$
\begin{aligned}
\pi_{j 1 \bullet} & =P\left(Z_{1}>z_{\bullet}, Z_{2}>z_{\bullet}\right) \geq P\left(Z_{1}>z_{\bullet}, Z_{2}>0\right) P\left(Z_{1}>0, Z_{2}>z_{\bullet}\right) \\
& =\pi_{j 2 。} \pi_{j 3}
\end{aligned}
$$

This is again always true if the expert's assessments are consistent with the bivariate exponential distribution. Sufficient conditions for having $t_{j 3} \geq \max \left(t_{j 1}, t_{j 2}\right)$ are:

$$
\begin{aligned}
& b_{j 3} \leq \min \left(b_{j 1}, b_{j 2}\right) \\
& \pi_{j 1,}^{2} \leq \pi_{j 2,} \pi_{j 3}, \min \left(\pi_{j 2,}, \pi_{j 3,}\right)
\end{aligned}
$$

Due to (2.7) the first of the conditions in (2.11) means that the jth expert assesses the prior mean of $\theta_{3}$ not less than the prior means of $\theta_{1}$ and $\theta_{2}$. The latter condition is true if the expert's assessments of the $\pi_{j v}$,'s are consistent with the bivariate exponential distribution having $\theta_{3} \leq \min \left(\theta_{1}, \theta_{2}\right)$, which in a way is the opposite condition. This makes sense since if the first condition is true the expert is allowed to be consistent by breaking the second one. If on the other hand the first one is not true, the expert is forced to be consistent by satisfying the second one.

Assume now that we have found $t_{j l}, j=1, \cdots, k ; l=1,2,3$ for instance by taking averages of the acceptable solutions to (2.10). Remember for the bivariate exponential distribution that:

$$
\begin{aligned}
& Z_{1 i}=\min \left(V_{1 i}, V_{3 i}\right) \\
& Z_{2 i}=\min \left(V_{2 i}, V_{3 i}\right),
\end{aligned}
$$

where the $V_{l i}$ 's are mutually independent and exponentially distributed with failure rates $\theta_{l}, l=1,2,3 ; i=1, \cdots, m$. The final aim of the DM is to compute his posterior distribution for $\left(Z_{1}, Z_{2}\right)$ given the information $t_{j l}, j=1, \cdots, k ; l=1,2,3$. When $B_{0}=\varnothing$, i.e. when the experts share no common information, this information is sufficient. Let $T_{j l}, j=$ $1, \cdots, k ; l=1,2,3$ be mutually independent and exponentially distributed with failure rates $\theta_{l}$. Then the DM calculates similar to (2.2):

$$
\begin{aligned}
& P\left[Z_{1}>z_{1}, Z_{2}>z_{2} \mid \bigcap_{j=1}^{k} \bigcap_{l=1}^{3}\left(T_{j l}>t_{j l}\right)\right] \\
& =\prod_{l=1}^{3}\left(\frac{b_{l}+\sum_{j=1}^{k} t_{j l}}{b_{l}+\sum_{j=1}^{k} t_{j l}+z_{l}}\right)^{a_{l}}
\end{aligned}
$$

This is nothing else than (2.2) since $\sum_{j=1}^{k} t_{j l}$ are the calculations of $t_{l}, l=1,2,3$ based on (2.10). 
When $B_{0} \neq \varnothing, t_{j l}, j=1, \cdots, k ; l=1,2,3$ is not sufficient information to perform our calculations. Let $T_{0 l}, l=1,2,3$ be mutually independent and exponentially distributed with failure rates $\theta_{l}, l=1,2,3$ and also independent of the $T_{j l}$ 's. Let us now assume that the $k$ experts agree to specify their common information as:

$$
\bigcap_{l=1}^{3}\left(T_{0 l}>c_{l}\right), \text { where } 0<c_{l}<t_{j l}, j=1, \cdots, k ; l=1,2,3
$$

The DM now has to calculate:

$$
\begin{aligned}
& P\left[Z_{1}>z_{1}, Z_{2}>z_{2} \mid \bigcap_{j=1}^{k} \bigcap_{l=1}^{3}\left(T_{0 l}+T_{j l}>t_{j l}\right) \bigcap \bigcap_{l=1}^{3}\left(T_{0 l}>c_{l}\right)\right] \\
& =k_{1} \int_{\theta_{1}=0}^{\infty} \int_{\theta_{2}=0}^{\infty} \int_{\theta_{3}=0}^{\infty} P\left(Z_{1}>z_{1}, Z_{2}>z_{2} \mid \theta_{1}, \theta_{2}, \theta_{3}\right) \\
& \int_{v_{1}=c_{1}}^{\infty} \int_{v_{2}=c_{2}}^{\infty} \int_{v_{3}=c_{3}}^{\infty} P\left[\bigcap_{j=1}^{k} \bigcap_{l=1}^{3}\left(T_{0 l}+T_{j l}>t_{j l}\right) \mid \bigcap_{l=1}^{3}\left(T_{0 l}=v_{l}\right), \theta_{1}, \theta_{2}, \theta_{3}\right] \\
& \prod_{l=1}^{3} \theta_{l} \exp \left(-v_{l} \theta_{l}\right) d v_{1} d v_{2} d v_{3} \prod_{l=1}^{3} \frac{b_{l}^{a_{l}} \theta_{l}^{a_{l}-1}}{\Gamma\left(a_{l}\right)} \exp \left(-b_{l} \theta_{l}\right) d \theta_{1} d \theta_{2} d \theta_{3} \\
& =k_{1} \int_{\theta_{1}=0}^{\infty} \int_{\theta_{2}=0}^{\infty} \int_{\theta_{3}=0}^{\infty} \int_{v_{1}=c_{1}}^{\infty} \int_{v_{2}=c_{2}}^{\infty} \int_{v_{3}=c_{3}}^{\infty} \prod_{j=1}^{k} \prod_{l=1}^{3} \theta_{l} \exp \left(-\left(v_{l}+\max \left(t_{j l}-v_{l}, 0\right)\right) \theta_{l}\right) d v_{1} d v_{2} d v_{3} \\
& \prod_{l=1}^{3} \frac{b_{l}^{a_{l}} \theta_{l}^{a_{l}-1}}{\Gamma\left(a_{l}\right)} \exp \left(-\left(b_{l}+z_{l}\right) \theta_{l}\right) d \theta_{1} d \theta_{2} d \theta_{3} \\
& =k_{1} \int_{v_{1}=c_{1}}^{\infty} \int_{v_{2}=c_{2}}^{\infty} \int_{v_{3}=c_{3}}^{\infty} \prod_{l=1}^{3} \int_{\theta_{l}=0}^{\infty} \frac{b_{l}^{a_{l}} \theta_{l}^{a_{l}}}{\Gamma\left(a_{l}\right)} \\
& \exp \left(-\left(k v_{l}+\sum_{j=1}^{k} \max \left(t_{j l}-v_{l}, 0\right)+b_{l}+z_{l}\right) \theta_{l}\right) d \theta_{l} d v_{1} d v_{2} d v_{3} \\
& =k_{2} \prod_{l=1}^{3} \int_{v_{l}=c_{l}}^{\infty}\left[k v_{l}+\sum_{j=1}^{k} \max \left(t_{j l}-v_{l}, 0\right)+b_{l}+z_{l}\right]^{-\left(a_{l}+1\right)} d v_{l}
\end{aligned}
$$

Now for fixed $l=1,2,3$ let $t_{l}^{(j)}$ be the ordered version of $t_{j l}, j=1, \cdots, k$. By letting 
$t_{l}^{(0)}=c_{l}, t_{l}^{(k+1)}=\infty$, the probability above equals:

$$
\begin{aligned}
& =k_{2} \prod_{l=1}^{3} \sum_{i=1}^{k+1} \int_{v_{l}=t_{l}^{(i-1)}}^{t_{l}^{(i)}}\left[\sum_{j=i}^{k} t_{l}^{(j)}+b_{l}+z_{l}+(i-1) v_{l}\right]^{-\left(a_{l}+1\right)} d v_{l} \\
& =k_{2} \prod_{l=1}^{3}\left\{\left[\sum_{j=1}^{k} t_{j l}+b_{l}+z_{l}\right]^{-\left(a_{l}+1\right)}\left(t_{l}^{(1)}-c_{l}\right)\right. \\
& +\sum_{i=2}^{k+1} \frac{1}{a_{l}(i-1)}\left[\left[(i-2) t_{l}^{(i-1)}+\sum_{j=i-1}^{k} t_{l}^{(j)}+b_{l}+z_{l}\right]^{-a_{l}}\right. \\
& \left.\left.-\left[(i-1) t_{l}^{(i)}+\sum_{j=i}^{k} t_{l}^{(j)}+b_{l}+z_{l}\right]^{\left.-a_{l}\right]}\right\}\right\} \\
& =k_{2} \prod_{l=1}^{3}\left\{\left[\sum_{j=1}^{k} t_{j l}+b_{l}+z_{l}\right]^{-\left(a_{l}+1\right)}\left(t_{l}^{(1)}-c_{l}\right)\right. \\
& \left.+\frac{1}{a_{l}}\left[\left[\sum_{j=1}^{k} t_{j l}+b_{l}+z_{l}\right]^{-a_{l}}-\sum_{i=3}^{k+1} \frac{1}{(i-1)(i-2)}\left[(i-2) t_{l}^{(i-l)}+\sum_{j=i-1}^{k} t_{l}^{(j)}+b_{l}+z_{l}\right]^{-a_{l}}\right]\right\}
\end{aligned}
$$

Hence we have shown that the DM's final assessment now is given by:

$$
\begin{aligned}
& P\left[Z_{1}>z_{1}, Z_{2}>z_{2} \mid \bigcap_{j=1}^{k} \bigcap_{l=1}^{3}\left(T_{0 l}+T_{j l}>t_{j l}\right) \bigcap \bigcap_{l=1}^{3}\left(T_{0 l}>c_{l}\right)\right]= \\
& \prod_{l=1}^{3}\left\{\sum_{j=1}^{k} t_{j l}+b_{l}+z_{l}\right]^{-\left(a_{l}+1\right)}\left(t_{l}^{(1)}-c_{l}\right) \\
& \left.+\frac{1}{a_{l}}\left[\left[\sum_{j=1}^{k} t_{j l}+b_{l}+z_{l}\right]^{-a_{l}}-\sum_{i=3}^{k+1} \frac{1}{(i-1)(i-2)}\left[(i-2) t_{l}^{(i-1)}+\sum_{j=i-1}^{k} t_{l}^{(j)}+b_{l}+z_{l}\right]^{\left.-a_{l}\right]}\right\}\right] \\
& \left\{\left[\sum_{j=1}^{k} t_{j l}+b_{l}\right]^{-\left(a_{l}+1\right)}\left(t_{l}^{(1)}-c_{l}\right)+\frac{1}{a_{l}}\left[\left[\sum_{j=1}^{k} t_{j l}+b_{l}\right]^{-a_{l}}-\sum_{i=3}^{k+1} \frac{1}{(i-1)(i-2)}\left[(i-2) t_{l}^{(i-1)}\right.\right.\right. \\
& \left.\left.+\sum_{j=i-1}^{k} t_{l}^{(j)}+b_{l}\right]^{\left.-a_{l}\right]}\right\}
\end{aligned}
$$

\section{The retrospective approach}

The deductions in this section follow the main lines of the example given in Section 5 of Huseby (1986). The jump from the univariate to the bivariate case is much easier than for 
the predictive approach treated in the previous section. Let $r_{l}, l=1,2,3$ and $p_{i}, i=1,2$ be defined as in (1.2). Also let $r_{4}=1-\sum_{l=1}^{3} r_{l}$. As in Huseby (1986) we let the number of experts $k$ be equal to 3 .

Assume that the information on the two components is given by the imaginary observations:

$$
\left(y_{i 1}, y_{i 2}, y_{i 3}\right), \quad i=1,2,3 \text {. }
$$

The DM assesses that:

$$
\left(y_{i 1}, y_{i 2}, y_{i 3} \mid r_{l}, l=1,2,3\right) \sim \text { Multinomial }\left(n_{i} ; r_{1}, r_{2}, r_{3}\right),
$$

and mutually independent, $i=1,2,3$. The jth expert has access to the observations:

$$
\left(y_{i 1}, y_{i 2}, y_{i 3}\right), i \in A_{j}=\{1,2,3\}-\{j\}, j=1,2,3 \text {. }
$$

However, their assessments are noisy, so instead of observing the "correct" values of the $y_{i l}$ 's, the jth expert observes:

$$
\left(z_{j i 1}, z_{j i 2}, z_{j i 3}\right), i \epsilon A_{j}, j=1,2,3 .
$$

The DM assumes that the jth expert assesses that:

$$
\left(z_{j i 1} z_{j i 2}, z_{j i 3} \mid r_{l}, l=1,2,3\right) \sim \text { Multinomial }\left(n_{i} ; r_{1}, r_{2}, r_{3}\right),
$$

and mutually independent, $i \epsilon A_{j}, j=1,2,3$. Finally, the DM assesses that the common prior of the experts, $g_{0}\left(r_{1}, r_{2}, r_{3}\right)$, is a density function of a Dirichlet-distribution with parameters close to zero.

Introduce the following notation $(i=1,2,3 ; j=1,2,3 ; l=1,2,3,4)$ :

$$
\begin{array}{rlrl}
y_{i 4} & =n_{i}-\sum_{l=1}^{3} y_{i l} & z_{j i 4} & =n_{i}-\sum_{l=1}^{3} z_{j i l} \\
m_{j} & =\sum_{i \in A_{j}} n_{i} \\
t_{j l} & =\sum_{i \in A_{j}} y_{i l} / m_{j} & z_{j l} & =\sum_{i \in A_{j}} z_{j i l} / m_{j}
\end{array}
$$

Then it is a standard result that the updated distribution for the jth expert is given by:

$$
\begin{aligned}
& g_{j}\left(r_{1}, r_{2}, r_{3} \mid z_{j l}, l=1,2,3\right) \\
& \sim \operatorname{Dirichlet}\left(r_{1}, r_{2}, r_{3} ; m_{j} z_{j 1}, m_{j} z_{j 2}, m_{j} z_{j 3}, m_{j} z_{j 4}\right)
\end{aligned}
$$

The Bayes sufficient statistic of the data $\left(y_{i l}\right), l=1,2,3 ; i=1,2,3$ is obviously:

$$
\underline{s}=\left(s_{1}, s_{2}, s_{3}\right),
$$


where we define $s_{l}=\sum_{i=1}^{3} y_{i l}, l=1,2,3,4$. Moreover, assume that the DM's prior distribution for $r_{1}, r_{2}, r_{3}$ is Dirichlet $\left(r_{1}, r_{2}, r_{3} ; \alpha_{1}, \alpha_{2}, \alpha_{3}, \alpha_{4}\right)$. It then follows that his updated distribution is given by:

$$
g\left(r_{1}, r_{2}, r_{3} \mid s\right) \sim \operatorname{Dirichlet}\left(r_{1}, r_{2}, r_{3} ; \alpha_{1}+s_{1}, \alpha_{2}+s_{2}, \alpha_{3}+s_{3}, \alpha_{4}+s_{4}\right)
$$

It remains to compute the DM's uncertainty about $s$ after having observed the $g_{j}$ 's from the experts given by (3.3), i.e. $f(\underline{s} \mid \underline{z})$ where:

$$
\underline{z}=\left(z_{11}, z_{12}, z_{13}, z_{21}, z_{22}, z_{23}, z_{31}, z_{32}, z_{33}\right)
$$

To do this the DM must specify the distribution of $\underline{z}$ given:

$$
\underline{y}=\left(y_{11}, y_{12}, y_{13}, y_{21}, y_{22}, y_{23}, y_{31}, y_{32}, y_{33}\right)
$$

and $r_{l}, l=1,2,3$. We assume that the DM assesses that

$$
\underline{z}_{j}=\left(z_{j 1}, z_{j 2}, z_{j 3}\right) \quad j=1,2,3
$$

are mutually independent given $\underline{y}, r_{l}, l=1,2,3$ and that in particular

$$
\begin{aligned}
& f_{j}\left(\underline{z}_{j} \mid \underline{y} ; r_{l}, l=1,2,3\right) \\
& \sim \operatorname{Dirichlet}\left(z_{j 1}, z_{j 2}, z_{j 3} ; \lambda_{j} t_{j 1}+\epsilon, \lambda_{j} t_{j 2}+\epsilon, \lambda_{j} t_{j 3}+\epsilon, \lambda_{j} t_{j 4}+\epsilon\right)
\end{aligned}
$$

where $\epsilon, \lambda_{1}, \lambda_{2}, \lambda_{3}$ are positive numbers specified by the DM. As in Huseby (1986) to get a better impression of this model, we set up the means, variances and covariances of this distribution. For $j=1,2,3$ these are given by:

$$
\begin{aligned}
& E\left(z_{j l} \mid \underline{y} ; r_{l}, l=1,2,3\right)=\frac{t_{j l}+\epsilon / \lambda_{j}}{1+4 \epsilon / \lambda_{j}}, \\
& l=1,2,3 \\
& \operatorname{Var}\left(z_{j l} \mid \underline{y} ; r_{l}, l=1,2,3\right)=\frac{\left(t_{j l}+\epsilon / \lambda_{j}\right)\left(1-t_{j l}+3 \epsilon / \lambda_{j}\right)}{\lambda_{j}\left(1+4 \epsilon / \lambda_{j}\right)^{2}\left(1+(4 \epsilon+1) / \lambda_{j}\right)}, \\
& l=1,2,3 \\
& \operatorname{Cov}\left(z_{j l}, z_{j v} \mid \underline{y} ; r_{l}, l=1,2,3\right)=\frac{\left(t_{j l}+\epsilon / \lambda_{j}\right)\left(t_{j v}+\epsilon / \lambda_{j}\right)}{\lambda_{j}\left(1+4 \epsilon / \lambda_{j}\right)^{2}\left(1+(4 \epsilon+1) / \lambda_{j}\right)}, \\
& l=1,2,3 ; v=1,2,3, l \neq v
\end{aligned}
$$

We observe that if $\lambda_{j} \rightarrow \infty$, then

$$
\begin{aligned}
& E\left(z_{j l} \mid \underline{y} ; r_{l}, l=1,2,3\right) \rightarrow t_{j l} \\
& \operatorname{Var}\left(z_{j l} \mid \underline{y} ; r_{l}, l=1,2,3\right) \rightarrow 0 \\
& \operatorname{Cov}\left(z_{j l}, z_{j v} \mid \underline{y} ; r_{l}, l=1,2,3\right) \rightarrow 0 .
\end{aligned}
$$


Hence the $z_{j l}$ 's are consistent and asymptotically unbiased estimators for the $t_{j l}$ 's as the $\lambda_{j}$ 's increase. Especially, if $\epsilon<<\lambda_{j}, j=1,2,3$ the bias is negligible. The term $\epsilon$ is included to ensure that the distribution $f_{j}\left(z_{j} \mid \underline{y} ; r_{l}, l=1,2,3\right)$ exists for all possible values of the $t_{j l}$ 's. Note that the distributions are independent of $r_{l}, l=1,2,3$.

In order to derive $f(\underline{s} \mid z)$, we first have to find $f(\underline{y} \mid z)$. This follows from Bayes' theorem:

$$
f(\underline{y} \mid z) \propto f(\underline{z} \mid \underline{y}) f(\underline{y})
$$

The first factor follows easily, since from (3.5):

$$
\begin{aligned}
& f(z \mid y)=f\left(z \mid y ; r_{l}, l=1,2,3\right) \\
& =\prod_{j=1}^{3} \Gamma\left(\lambda_{j}+4 \epsilon\right) \prod_{l=1}^{1} \frac{z_{j l}^{\lambda_{j} t_{j l}+\epsilon-1}}{\Gamma\left(\lambda_{j} t_{j l}+\epsilon\right)}
\end{aligned}
$$

Concerning the second factor, we obtain from (3.1):

$$
\begin{aligned}
& f(\underline{y})=\int_{r_{1}=0}^{1} \int_{r_{2}=0}^{1} \int_{r_{3}=0}^{1} f\left(\underline{y} \mid r_{1}, r_{2}, r_{3}\right) f\left(r_{1}, r_{2}, r_{3}\right) d r_{1} d r_{2} d r_{3} \\
& =\int_{r_{1}=0}^{1} \int_{r_{2}=0}^{1} \int_{r_{3}=0}^{1} \prod_{i=1}^{3} n_{i} ! \prod_{l=1}^{4} \frac{r_{l}^{y_{i}}}{y_{i l} !} \Gamma\left(\alpha_{1}+\alpha_{2}+\alpha_{3}+\alpha_{4}\right) \prod_{s=1}^{4} \frac{r_{0}^{\alpha_{0}-1}}{\Gamma\left(\alpha_{3}\right)} d r_{1} d r_{2} d r_{3} \\
& =\frac{\Gamma\left(\alpha_{1}+\alpha_{2}+\alpha_{3}+\alpha_{4}\right)}{\Gamma\left(n_{1}+n_{2}+n_{3}+\alpha_{1}+\alpha_{2}+\alpha_{3}+\alpha_{4}\right)} \prod_{l=1}^{1} \frac{\Gamma\left(s_{l}+\alpha_{l}\right)}{\Gamma\left(\alpha_{l}\right)} \prod_{i=1}^{3} \frac{n_{i} !}{y_{i l} !}
\end{aligned}
$$

By combining (3.7) and (3.8) we get from (3.6):

$$
f(\underline{y} \mid \underline{z}) \propto \frac{\Gamma\left(\alpha_{1}+\alpha_{2}+\alpha_{3}+\alpha_{4}\right)}{\Gamma\left(n_{1}+n_{2}+n_{3}+\alpha_{1}+\alpha_{2}+\alpha_{3}+\alpha_{4}\right)} \prod_{i=1}^{3} n_{i} ! \Gamma\left(\lambda_{i}+4 \epsilon\right) \prod_{l=1}^{4} \frac{z_{i l}^{\lambda_{i} t_{i l}+\epsilon-1} \Gamma\left(s_{l}+\alpha_{l}\right)}{\Gamma\left(\lambda_{i} t_{i l}+\epsilon\right) \Gamma\left(\alpha_{l}\right) y_{i l} !}
$$

By denoting:

$$
Y_{\underline{\underline{a}}}=\{\underline{y} \mid \underline{s}(\underline{y})=\underline{s}\}
$$

we get:

$$
f(\underline{s} \mid \underline{z})=\sum_{\underline{\underline{y}} \in Y_{\underline{\underline{a}}}} f(\underline{y} \mid \underline{z})
$$

Introducing

$$
S=\left\{\underline{s} \mid 0 \leq s_{1}+s_{2}+s_{3} \leq n_{1}+n_{2}+n_{3}\right\},
$$

from (3.4), (3.8) and (3.9) we finally end up with:

$$
\begin{aligned}
& g\left(r_{1}, r_{2}, r_{3} \mid z\right)=\sum_{S} g\left(r_{1}, r_{2}, r_{3} \mid \underline{s}\right) f(\underline{s} \mid z) \\
& \propto \sum_{S} A_{\underline{g}} \operatorname{Dirichlet}\left(r_{1}, r_{2}, r_{3} ; \alpha_{1}+s_{1}, \alpha_{2}+s_{2}, \alpha_{3}+s_{3}, \alpha_{4}+s_{4}\right),
\end{aligned}
$$


where

$$
A_{\underline{g}}=\sum_{\underline{y} \epsilon Y_{\underline{b}}} \frac{\Gamma\left(\alpha_{1}+\alpha_{2}+\alpha_{3}+\alpha_{4}\right)}{\Gamma\left(n_{1}+n_{2}+n_{3}+\alpha_{1}+\alpha_{2}+\alpha_{3}+\alpha_{4}\right)} \prod_{i=1}^{3} n_{i} ! \Gamma\left(\lambda_{i}+4 \epsilon\right) \prod_{l=1}^{4} \frac{z_{i l}^{\lambda_{i} t_{i l}+\epsilon-1} \Gamma\left(s_{l}+\alpha_{l}\right)}{\Gamma\left(\lambda_{i} t_{i l}+\epsilon\right) \Gamma\left(\alpha_{l}\right) y_{i l} !}
$$

Hence $g\left(r_{1}, r_{2}, r_{3} \mid \underline{z}\right)$ is a weighted average of Dirichlet distributions.

The joint distribution of the DM's assessment of the reliabilities of the two components, given the opinions of the experts, is then from (1.2) and (3.10):

$$
\begin{aligned}
& \pi\left(p_{1}, p_{2} \mid z\right)=\int_{r_{3}=0}^{\min \left(p_{1}, p_{2}\right)} g\left(p_{1}-r_{3}, p_{2}-r_{3}, r_{3} \mid z\right) d r_{3} \\
& \propto \sum_{S} B_{3} \int_{r_{3}=0}^{\min \left(p_{1}, p_{2}\right)}\left(p_{1}-r_{3}\right)^{\alpha_{1}+s_{1}-1}\left(p_{2}-r_{3}\right)^{\alpha_{2}+s_{2}-1} r_{3}^{\alpha_{3}+s_{3}-1} \\
& \left(1-p_{1}-p_{2}+r_{3}\right)^{\alpha_{4}+s_{4}-1} d r_{3},
\end{aligned}
$$

where

$$
B_{\underline{s}}=A_{\underline{g}} \Gamma\left(n_{1}+n_{2}+n_{3}+\alpha_{1}+\alpha_{2}+\alpha_{3}+\alpha_{4}\right) \prod_{l=1}^{4} \frac{1}{\Gamma\left(s_{l}+\alpha_{l}\right)} .
$$

Introducing $\beta_{l}=\alpha_{l}+s_{l}-1, l=1,2,3,4$, the integral above, $I$, can be written as:

$$
\begin{aligned}
I & =\int_{0}^{\min \left(p_{1}, p_{2}\right)}\left(p_{1}-x\right)^{\beta_{1}}\left(p_{2}-x\right)^{\beta_{2}} x^{\beta_{3}}\left(1-p_{1}-p_{2}+x\right)^{\beta_{4}} d x \\
& =\int_{0}^{\min \left(p_{1}, p_{2}\right)} \sum_{i=0}^{\beta_{1}}\left(\begin{array}{c}
\beta_{1} \\
i
\end{array}\right) p_{1}^{i}(-x)^{\beta_{1}-i} \sum_{j=0}^{\beta_{2}}\left(\begin{array}{c}
\beta_{2} \\
j
\end{array}\right) p_{2}^{j}(-x)^{\beta_{2}-j} x^{\beta_{3}} \\
& \sum_{k=0}^{\beta_{4}}\left(\begin{array}{c}
\beta_{4} \\
k
\end{array}\right)\left(1-p_{1}-p_{2}\right)^{k} x^{\beta_{4}-k} d x \\
& =\sum_{i=0}^{\beta_{1}} \sum_{j=0}^{\beta_{2}} \sum_{k=0}^{\beta_{4}}\left(\begin{array}{c}
\beta_{1} \\
i
\end{array}\right)\left(\begin{array}{c}
\beta_{2} \\
j
\end{array}\right)\left(\begin{array}{c}
\beta_{4} \\
k
\end{array}\right) p_{1}^{i} p_{2}^{j}\left(1-p_{1}-p_{2}\right)^{k}(-1)^{\beta_{1}+\beta_{2}-i-j} \\
& \min \left(p_{1}, p_{2}\right) \\
& \int_{0}^{\beta_{1}+\beta_{2}+\beta_{3}+\beta_{4}-(i+j+k)} d x
\end{aligned}
$$

Hence we have:

$$
\begin{aligned}
& \pi\left(p_{1}, p_{2} \mid \underline{z}\right) \propto \sum_{S} B_{\underline{s}} \sum_{i=0}^{\beta_{1}} \sum_{j=0}^{\beta_{2}} \sum_{k=0}^{\beta_{4}}\left(\begin{array}{c}
\beta_{1} \\
i
\end{array}\right)\left(\begin{array}{c}
\beta_{2} \\
j
\end{array}\right)\left(\begin{array}{c}
\beta_{4} \\
k
\end{array}\right) \\
& p_{1}^{i} p_{2}^{j}\left(1-p_{1}-p_{2}\right)^{k}(-1)^{\beta_{1}+\beta_{2}-i-j}\left(\min \left(p_{1}, p_{2}\right)\right)^{\beta_{1}+\beta_{2}+\beta_{3}+\beta_{4}+1-(i+j+k)} \\
& /\left(\beta_{1}+\beta_{2}+\beta_{3}+\beta_{4}+1-(i+j+k)\right)
\end{aligned}
$$




\section{The link to the paper Natvig and Eide (1987)}

In this section we will try to link our work on using expert opinions to the approach of Natvig and Eide (1987). We then start out with the joint prior distribution of the reliabilities, $\pi(\underline{p})$, arrived at as in the previous section. Let us first consider the case of independent components given $p$. Suppose that we run experiments on the component level and get the data $\underline{D}=\left(D_{1}, \cdots, D_{n}\right)$ where $D_{i}$ is the data from the experiment on the ith component. Let $\pi(\underline{D} \mid p)$ be the corresponding likelihood function. Hence the posterior distribution of the reliabilities, $\pi(\underline{p} \mid \underline{D})$, is given by:

$$
\pi(\underline{p} \mid \underline{D})=\frac{\pi(\underline{D} \mid \underline{p}) \pi(\underline{p})}{\int \pi(\underline{D} \mid \underline{p}) \pi(\underline{p}) d \underline{\underline{p}}}
$$

The corresponding distribution of system reliability $\pi\{h(p) \mid \underline{D}\}$ can in principle be arrived at by using the transformation formula for joint probability distributions. The prior dependencies between $p_{1}, \cdots, p_{n}$ are not creating too much extra trouble here. By now using expert opinion on the system level, in the spirit of Huseby (1986), $\pi\{h(\underline{p}) \mid \underline{D}\}$ may be updated to the prior distribution of system reliability $\pi_{0}\{h(p) \mid \underline{D}\}$. If we now finally run an experiment on the system level and get the data $D$, we end up with the posterior distribution of system reliability $\pi\{h(\underline{p} \mid \underline{D}, D\}$.

Let us next consider the case of associated components given $\underline{p}$. Then from (4.1) the marginal posterior distribution of $p_{i}, \pi\left(p_{i} \mid \underline{D}\right)$, is given by:

$$
\pi\left(p_{i} \mid \underline{D}\right)=\frac{\int \pi(\underline{D} \mid \underline{p}) \pi(\underline{p}) d(\cdot i, \underline{p})}{\int \pi(\underline{D} \mid \underline{p}) \pi(\underline{p}) d \underline{p}}
$$

where $\left(\cdot_{i}, \underline{p}\right)=\left(p_{1}, \cdots, p_{i-1}, \cdot p_{i+1}, \cdots, p_{n}\right)$. This leads to the moments up till order $m(i=$ $1, \cdots, n ; j=1, \cdots, m)$ :

$$
E\left(p_{i}^{j} \mid \underline{D}\right)=\frac{\int p_{i}^{j} \pi(\underline{D} \mid \underline{p}) \pi(\underline{p}) d \underline{p}}{\int \pi(\underline{D} \mid \underline{p}) \pi(\underline{p}) d \underline{\underline{p}}}
$$

by for instance applying the mentioned approximation technique suggested by Laplace. From (4.3) by applying results of Natvig and Eide (1987) we arrive at bounds on:

$$
E\left(h^{j} \underline{D}\right) \quad j=1, \cdots, m,
$$

of $\pi(h \underline{D})$.

However, the best bounds in the latter paper are based on the assumption that $p_{1}, \cdots, p_{n}$ are independent given $\underline{D}$. Sufficient conditions for this are that the components have independent prior reliabilities, which is unrealistic when the opinions of experts are used, and that $D_{1}, \cdots, D_{n}$ are independent given $p$, which is reasonable if for instance different laboratories are used for different components. From (4.4) one may adjust a proper $\pi(h \mid \underline{D})$, which may be further updated to $\pi_{0}(h \mid \underline{D})$ and $\pi(h \mid \underline{D}, D)$ as in the case of independent components. 
The rather good lower bounds of Theorem 2.7 of Natvig and Eide (1987) are valid also under the weaker assumption that $p_{1}, \cdots, p_{n}$ are associated given $\underline{D}$. From (4.1) and a fundamental inequality given in Corollary 4.1 of Karlin and Rinott (1980) a sufficient condition for this to be true is that $\pi(\underline{D} \mid \underline{p})$ and $\pi(\underline{p})$ both are $M T P_{2}$ (Multivariate Totally Positive of order 2) in $p$.

Note that a random vector $\left(Z_{1}, \cdots, Z_{n}\right)$ is $M T P_{2}$ if and only if its density, $f(z)$, is $M T P_{2}$, i.e. if:

$$
f(\underline{x} \vee \underline{y}) f(\underline{x} \wedge \underline{y}) \geq f(\underline{x}) f(\underline{y}),
$$

where for $\underline{x}=\left(x_{1}, \cdots, x_{n}\right), \underline{y}=\left(y_{1}, \cdots, y_{n}\right)$

$$
\begin{aligned}
& \underline{x} \vee \underline{y}=\left(\max \left(x_{1}, y_{1}\right), \cdots, \max \left(x_{n}, y_{n}\right)\right) \\
& \underline{x} \wedge \underline{y}=\left(\min \left(x_{1}, y_{1}\right), \cdots, \min \left(x_{n}, y_{n}\right)\right) .
\end{aligned}
$$

For the time being we have no idea of how the $M T P_{2}$ property can be converted into assumptions on observable random quantities. In the case where $D_{1}, \cdots, D_{n}$ are independent given $\underline{p}$, we have:

$$
\pi(\underline{D} \mid p)=\prod_{i=1}^{n} \pi\left(D_{i} \mid p_{i}\right),
$$

and it follows from Proposition 3.3 of Karlin and Rinott (1980) that $\pi(\underline{D} \mid \underline{p})$ is $M T P_{2}$. Hence a key question to be answered is the following. When does $\pi(p)$ established by using expert opinions in fact possess the $M T P_{2}$ property? According to Theorem 4.2 of Karlin and Rinott (1980) this property is stronger than the property of association.

For the case treated in Section 3 one may ask more specifically whether $\pi\left(p_{1}, p_{2} \mid \underline{z}\right)$ given by (3.11) is $M T P_{2}$. According to Proposition 3.2 and 3.3 of Karlin and Rinott (1980) a sufficient condition for this to be true is that each of the functions $\left(p_{1}-r_{3}\right),\left(p_{2}-r_{3}\right)$ and $\left(1-p_{1}-p_{2}+r_{3}\right)$ is $M T P_{2}$. It is easy to check that the first two functions in fact are $M T P_{2}$, but, alas, the last one is not.

The best we can do at the present stage of research is to consider the non trivial, but strongly dependent case, where $r_{2}=0$ with probability 1 . Then it is rather easy to check that (3.11) reduces to:

$$
\pi\left(p_{1}, p_{2} \mid z\right) \propto \sum_{S} B_{2}\left(p_{1}-p_{2}\right)^{\beta_{1}} p_{2}^{\beta_{3}}\left(1-p_{1}\right)^{\beta_{4}}
$$

where $\alpha_{2}=0$ in the expression for $B_{\underline{g}}$. It now follows from the latter proposition that $\pi\left(\left(p_{1}, p_{2} \mid \underline{z}\right)\right.$ is $M T P_{2}$. Of course the same holds when $r_{1}=0$ with probability 1 .

Consider now a parallel system of the two components in the case $r_{2}=0$ with probability 1. Then:

$$
\begin{aligned}
& h=E\left[1-\prod_{i=1}^{2}\left(1-X_{i}\right)\right]=E X_{1}+E X_{2}-E X_{1} X_{2} \\
& =p_{1}+p_{2}-r_{3}=p_{1}
\end{aligned}
$$


Hence:

$$
\begin{aligned}
& \pi(h \mid z)=\pi\left(p_{1} \mid z\right)=\int_{0}^{p_{1}} \pi\left(p_{1}, p_{2} \mid z\right) d p_{2} \\
& \propto \sum_{S} B_{2} p_{1}^{\beta_{1}+\beta_{2}+1}\left(1-p_{1}\right)^{\beta_{4}} \int_{0}^{1}(1-x)^{\beta_{1}} x^{\beta_{2}} d x \\
& =\sum_{S} B_{\underline{L}} \frac{\Gamma\left(\beta_{1}+1\right) \Gamma\left(\beta_{3}+1\right)}{\Gamma\left(\beta_{1}+\beta_{3}+2\right)} p_{1}^{\beta_{1}+\beta_{2}+1}\left(1-p_{1}\right)^{\beta_{4}}
\end{aligned}
$$

Correspondingly for a series system in this case:

$$
\begin{aligned}
& \pi(h \mid \underline{z})=\pi\left(p_{2} \mid \underline{z}\right)=\int_{p_{2}}^{1} \pi\left(p_{1}, p_{2} \mid z\right) d p_{1} \\
& =\sum_{S} B_{3} p_{2}^{\beta_{3}} \int_{p_{2}}^{1} \sum_{i=0}^{\beta_{1}}\left(\begin{array}{c}
\beta_{1} \\
i
\end{array}\right) p_{1}^{\beta_{1}-i}\left(-p_{2}\right)^{i} \sum_{j=0}^{\beta_{4}}\left(\begin{array}{c}
\beta_{1} \\
j
\end{array}\right)\left(-p_{1}\right)^{j} d p_{1} \\
& =\sum_{S} B_{-} \sum_{i=0}^{\beta_{1}} \sum_{j=0}^{\beta_{4}}\left(\begin{array}{c}
\beta_{1} \\
i
\end{array}\right)\left(\begin{array}{c}
\beta_{4} \\
j
\end{array}\right)(-1)^{i+j} \frac{1}{\beta_{1}+j-i+1}\left(p_{2}^{\beta_{3}+i}-p_{2}^{\beta_{1}+\beta_{3}+j+1}\right) .
\end{aligned}
$$

\section{Conclusions}

It seems that generalizing Sections 2 and 3 to the case $n>3$ is more a matter of stamina than of new ideas. Concerning the $M T P_{2}$ connection things are less obvious and new ideas seem very welcome. A paper at least worth mentioning is Fahmy et al. (1982) being interested in the influence of the sample on the posterior distribution.

\section{References}

Barlow, R.E. and Proschan, F. (1975) Statistical theory of reliability and life testing. Probability models. Holt, Rinehart and Winston, New York.

Fahmy,S., Pereira, C.A., Proschan, F. and Shaked, M. (1982) The influence of the sample on the posterior distribution. Commun. Statist. A, 11, 1757-1768.

Huseby, A.B. (1986) Combining experts' opinions, a retrospective approach. Tech. Rep. Center for Industrial Research, P.O. Box 350, Blindern, Oslo 3, Norway.

Huseby, A.B. (1988) Combining opinions in a predictive case. In Bayesian Statistics 3. Eds. Bernardo, J.M, DeGroot M.H., Lindley, D.V. and Smith, A.F.M. Oxford University Press, 641-651. 
Karlin, S. and Rinott, Y. (1980) Classes of orderings of measures and related correlation inequalities. I. Multivariate totally positive distributions. J. Multivariate Analysis, 10, 467-498.

Lindley, D.V. and Singpurwalla, N.D. (1986) Reliability (and fault tree) analysis using expert opinions. J. Amer. Statist. Ass., 81, 87-90.

Natvig, B. and Eide, H. (1987) Bayesian estimation of system reliability. Scand. J. Statist., 14, 319-327.

Tierney, L. and Kadane, J.B. (1986) Accurate approximations for posterior moments and marginal densities. J. Amer. Statist. Ass., 81, 82-86. 